\title{
Prototyping of a multicell superconducting cavity for acceleration of medium-velocity beams
}

\author{
C. C. Compton, T. L. Grimm, W. Hartung, H. Podlech, ${ }^{*}$ and R. C. York \\ National Superconducting Cyclotron Laboratory, Michigan State University, East Lansing, Michigan 48824, USA \\ G. Ciovati and P. Kneisel \\ Thomas Jefferson National Accelerator Facility, Newport News, Virginia 23606, USA \\ D. Barni, C. Pagani, and P. Pierini \\ INFN Milano-Laboratorio LASA, 20090 Segrate (Milano), Italy
}

(Received 24 June 2004; published 11 April 2005)

\begin{abstract}
Three 6-cell $805 \mathrm{MHz}$ superconducting cavity prototypes for acceleration in the velocity range of about 0.4 to 0.53 times the speed of light have been fabricated and tested. The quality factors $\left(Q_{0}\right)$ were between $7 \times 10^{9}$ and $1.4 \times 10^{10}$ at the design field (accelerating gradient of $8-10 \mathrm{MV} / \mathrm{m}$ ). The maximum gradients reached were between 11 and $16 \mathrm{MV} / \mathrm{m}$; in each case, the $Q_{0}$ values were $\geq 3 \times 10^{9}$ at the maximum gradient. The design, fabrication, surface preparation, and rf testing of the 6-cell cavities are reported in this paper.
\end{abstract}

DOI: $10.1103 /$ PhysRevSTAB.8.042003

\section{INTRODUCTION}

Superconducting accelerators are presently used to accelerate particles that are light enough to have $v / c \equiv \beta \approx$ 1 (electrons and positrons) or heavy enough (ions) to have $\beta \ll 1$ (where $v$ is the beam velocity and $c$ is the speed of light). Historically, superconducting cavities have not been used in between these two extremes. A bridge is now being constructed to span this gap: in recent years, a number of superconducting accelerators have been proposed to accelerate particles all the way from very low $\beta$ to $\beta$ close to 1 .

To achieve this, the traditional low- $\beta$ structures (quarterwave resonators and variants thereof, typically used for $\beta \leq 0.2$ ) are being doubled up (into half-wave resonators and variants thereof) to extend their reach to higher $\beta$ values. Likewise, the traditional $\beta=1$ structures (axisymmetric cavities of elliptical cross section) are being shortened to reach to lower $\beta$ values.

The latter approach was pioneered at Los Alamos, where the original goal was a $1 \mathrm{GeV}$ pion linac, PILAC [1]. It was realized from the beginning that it is better to use a few different cavity shapes, each optimized for one specific $\beta$ value, rather than making each cavity in the accelerator unique to the exact $\beta$ of the beam at the location of the cavity; the advantages of simplified cavity fabrication and increased flexibility for installation and operation more than make up for the fact that the cavity voltages are not used with complete efficiency everywhere along the accelerator.

The PILAC concept did not bear fruit, but the buds grown at Los Alamos were grafted onto other branches of the tree of particle accelerators. A number of lower- $\beta$

\footnotetext{
*Present address: Institut für Angewandte Physik, GoetheUniversität, Frankfurt am Main, Germany.
}

PACS numbers: 84.40. $-\mathrm{x}, 85.25 . \mathrm{Am}, 52.70 . \mathrm{Gw}, 29.17 .+\mathrm{w}$

elliptical cavity prototypes have now been built in France, Germany, Italy, Japan, and the United States [2-10]. Moreover, the Spallation Neutron Source (SNS), presently under construction, adopted superconducting elliptical cavities with $\beta=0.61$ and $\beta=0.81$ for the SNS linac. The successful results with prototype multicell cavities for SNS [11], as well as several other projects [6-9], provide a good basis for this technology choice.

Though SNS will use normal conducting cavities for velocities below the reach of the SNS $\beta=0.61$ cavity, single-cell prototypes of elliptical cavities for even lower $\beta$ values have been prototyped at several laboratories: two $\beta=0.48$ cavities $(700 \mathrm{MHz}$ ) were tested by Los Alamos [2]; a $\beta=0.45$ cavity (1.3 GHz) was tested by KEK [3]; two $\beta=0.5$ cavities $(600 \mathrm{MHz})$ were tested by JAERI [4]; a $\beta=0.47$ cavity $(700 \mathrm{MHz})$ was tested by INFN-Milano and Saclay [10]. Encouragingly, the desired gradients and quality factors were exceeded in all of these single-cell tests.

A 5 -cell $\beta=0.5$ cavity prototype $(600 \mathrm{MHz})$ was also tested by JAERI and KEK [7]. Good low-field performance was ultimately achieved [12]. The high-field performance was not as good as expected, but this was not attributed to anything intrinsic to the low $\beta$.

In light of the promising results with elliptical cavities for $\beta$ in the vicinity of 0.5 , a medium- $\beta$ multicell cavity development program was undertaken as a collaboration between INFN-Milano, Jefferson Lab, and Michigan State University (MSU). The work is being carried out as part of the design of the driver linac for the proposed Rare Isotope Accelerator (RIA). RIA is being designed to provide an intense beam of exotic isotopes for nuclear physics research [13]. The driver linac is to accelerate heavy ions to high energy (400 MeV per nucleon or higher), with a high beam power (up to $400 \mathrm{~kW}$ ). In order to maximize the 
beam power, the driver linac will operate continuously, making it advantageous for the entire linac to be superconducting [14]. Quarter-wave and half-wave resonators are to be used for the low- $\beta$ portion of the linac; elliptical cavities, including the $\beta=0.61$ and $\beta=0.81$ SNS cavities, are to be used for the high-energy portion. Though the present development effort is for RIA, the $\beta=0.47$ technology could also be applied to superconducting linacs for proton or $\mathrm{H}^{-}$beams, several of which have been proposed $[15,16]$.

This paper covers the design of the 6-cell $\beta=0.47$ cavity, the fabrication of prototype cavities, and rf tests on two single-cell prototypes and three multicell prototypes. More complete information on the single-cell results may be found in the literature $[17,18]$. Preliminary results on the multicell cavities have also been previously reported [19].

\section{CAVITY DESIGN}

Let us define the maximum $\beta \equiv \beta_{m}$ to be the $\beta$ value for which the beam receives the maximum accelerating kick from the actual cavity, and the geometric $\beta \equiv \beta_{g}$ to be the maximum $\beta$ value in the case of a periodic structure, i.e., a multicell structure in the limit of an arbitrarily large number of cells. For obvious reasons, the latter quantity is usually referred to for high- $\beta$ cavities, while the former quantity (also called "optimum $\beta$ ") is usually given for low- $\beta$ cavities.

The SNS $\beta_{g}=0.81$ and $\beta_{g}=0.61$ cavities are the basis for our $\beta_{g}=0.47$ cell shape $[17,20]$. The $\beta_{g}=$ 0.47 cell shape is compared with those of the SNS cavities and the TESLA Test Facility (TTF) cavity [21] in Fig. 1. As can be seen, the $\beta_{g}=0.47$ cell shape is relatively close to

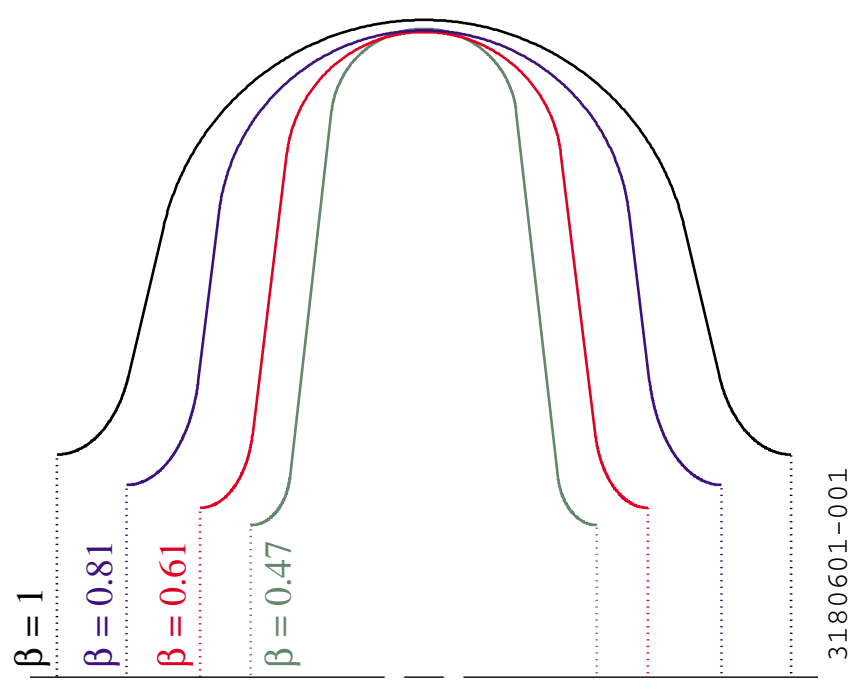

FIG. 1. (Color) Comparison of the $\beta_{g}=0.47$ cell with a $\beta_{g}=$ 0.61 cell (SNS), a $\beta_{g}=0.81$ cell (SNS), and a $\beta_{g}=1$ cell (TTF, scaled to $805 \mathrm{MHz}$ ). the $\beta_{g}=0.61$ cell shape. Thus, stiffening and bracing techniques being used for the SNS cavities can be applied to the $\beta_{g}=0.47$ cavity also.

As indicated above, medium- $\beta$ cavities must accelerate a beam whose velocity is varying with position. The penalty due to the velocity mismatch can be quantified via the transit time factor (the maximum energy gain for a particle traveling at speed $\beta c$ divided by the energy gain for the ideal case in which the field's time variation is not accounted for and the phase is optimal at every point along the particle's path). The transit time factor of the $\beta_{g}=$ 0.47 cavity is shown in Fig. 2 for the 6-cell case and two other cases. The transit time factor was calculated with SUPERFISH [22,23].

The approximate range of usefulness of the 6-cell cavity is from $\beta=0.4$ to $\beta=0.53$ (indicated by dotted lines in Fig. 2). The useful range is not determined by the cavity characteristics alone; for RIA as an example, different cavity geometries are used upstream and downstream of the $\beta_{g}=0.47$ cavities, and the useful velocity range for each cavity is determined from the velocities at which one cavity geometry becomes more efficient than another.

The choice of 6 cells is a reasonable compromise between a long structure to provide higher voltage and a small number of cells for higher velocity acceptance. Note that $\beta_{m}$ is between 0.49 and 0.50 in the 6-cell case, i.e., slightly larger than $\beta_{g}$; particles with $\beta$ slightly larger than $\beta_{g}$ acquire a little more energy traveling through the cavity and suffer less deceleration from the evanescent field in the beam tube. As can be seen in Fig. 2, the discrepancy between $\beta_{g}$ and $\beta_{m}$ becomes more pronounced as the number of cells is reduced.

The beam tube is enlarged on one side of the SNS cavities to provide stronger input coupling. Less coupling is needed for RIA, so no enlargement of the beam tube is

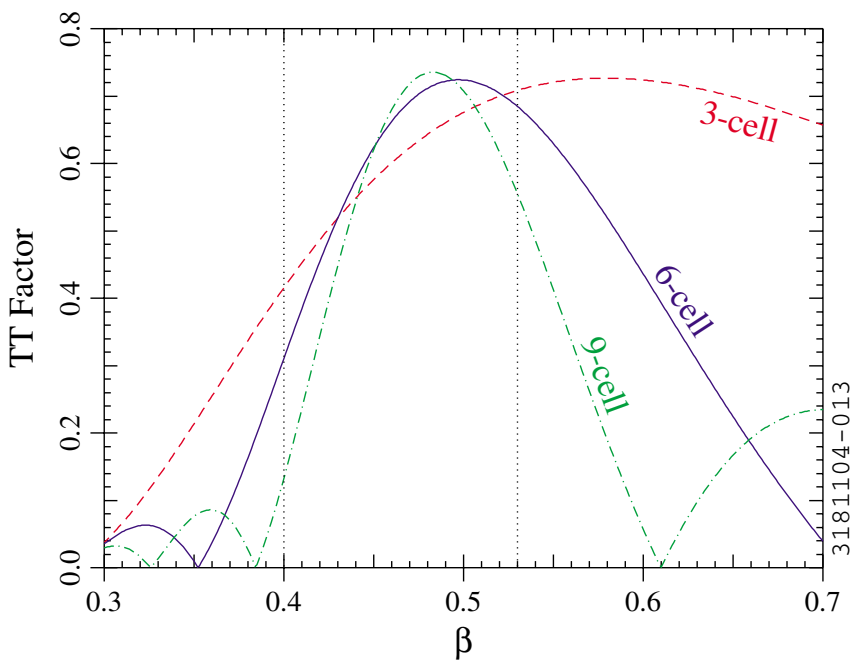

FIG. 2. (Color) Dependence of the transit time factor on $\beta$ for the $\beta_{g}=0.47$ cavity for 3,6 , and 9 cells. 
TABLE I. Parameters of the symmetric 6-cell $\beta_{g}=0.47$ cavity. The rf quantities were calculated with SUPERFISH.

\section{Cell mode}

Phase advance per cell

Resonant frequency $f$

Cell-to-cell coupling $\equiv 2\left(f_{\pi}-f_{0}\right) /\left(f_{\pi}+f_{0}\right)$

$E_{p} / E_{a}$

$c B_{p} / E_{a}$

$R_{a} / Q$

Geometry factor $G$

$\mathrm{TM}_{010}$
$\pi$
$805 \mathrm{MHz}$
$1.5 \%$
3.34
1.98
$173 \Omega$
$136 \Omega$
$527 \mathrm{~mm}$
$77.2 \mathrm{~mm}$
$329 \mathrm{~mm}$

required for the $\beta_{g}=0.47$ cavity [24]. This simplifies the cavity fabrication and yields a slight improvement in the rf parameters of the structure. Selected cavity parameters are given in Table I. The accelerating mode is a standing-wave mode, as is the case for most superconducting cavities. In Table I, $E_{p}$ and $B_{p}$ are the peak surface electric and magnetic field, respectively; $R_{a}$ is the shunt impedance (linac definition) and $E_{a}$ is the accelerating gradient (both include the transit time factor with $\beta=\beta_{g}$ ). The rf parameters in Table I were calculated with SUPERFISH [22,23] and checked with SUPERLANS [25].

An analysis was done of the excitation of higher-order modes (HOMs) in the cavity by the beam and coupling of the HOMs to the input coupler and pickup antenna. This analysis indicates that HOM couplers are not required for operation of the $\beta_{g}=0.47$ cavity in RIA, allowing for further simplification of the system [24].

\section{SINGLE-CELL CAVITY PROTOTYPING}

Two single-cell prototypes of the $\beta_{g}=0.47$ cavity were fabricated and tested. Some of the results are shown in Fig. 3. The highest gradient reached in the first round of tests [17] was about $15 \mathrm{MV} / \mathrm{m}$. As can be seen in Fig. 3, the $Q_{0}$ values at $15 \mathrm{MV} / \mathrm{m}$ were about $10^{10}$; the low-field $Q_{0}$ values were between $2 \times 10^{10}$ and $4 \times 10^{10}$. These measurements were done at $2 \mathrm{~K}$ in a vertical cryostat at Jefferson Lab.

A number of additional tests were done on the second of the two single-cell cavities while commissioning the facilities at MSU for etching, high-pressure rinsing, clean assembly, rf testing, and helium processing of superconducting cavities. The highest gradient reached in these tests was about $18 \mathrm{MV} / \mathrm{m}$, albeit with a slightly lower $Q_{0}$; nevertheless, $Q_{0}$ still exceeded $10^{10}$ at $E_{a}=10 \mathrm{MV} / \mathrm{m}$ [18]. As can be seen in Fig. 3, some improvement in the low-field $Q_{0}$ was seen at temperatures below $2 \mathrm{~K}$, but the high-field performance did not change much.

More detailed information on the single-cell results may be found elsewhere $[17,18]$.

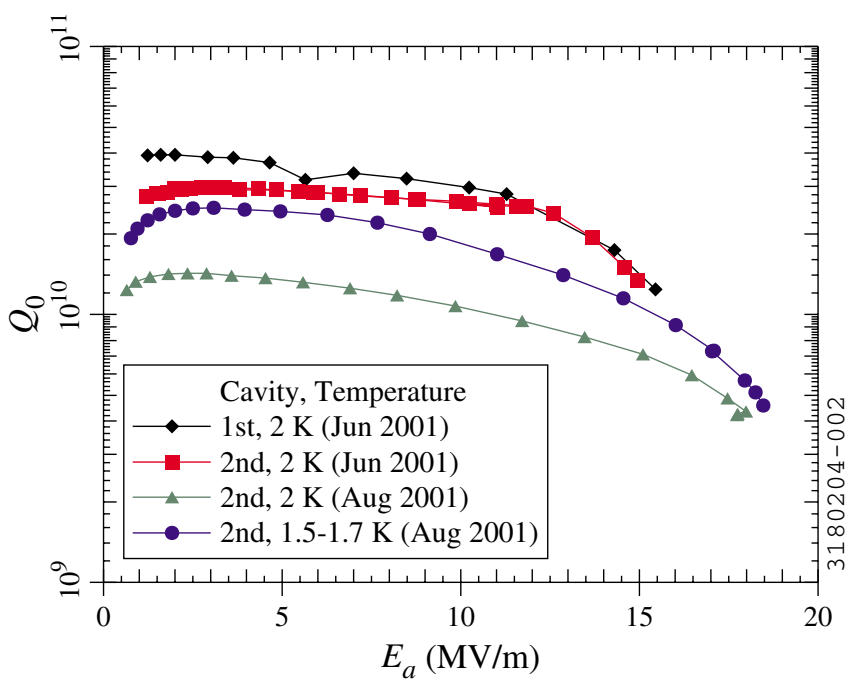

FIG. 3. (Color) Measured dependence of the quality factor on the accelerating gradient for the single-cell cavity prototypes. The June 2001 measurements were done at Jefferson Lab; the August 2001 measurements were done at MSU. In the June 2001 tests, there may have been some rf conditioning during the measurements, as evidenced by a slight decrease and recovery in $Q_{0}$ at 6-7 MV/m (1st cavity) or 11-12 MV/m (2nd cavity).

\section{MULTICELL CAVITY PROTOTYPING}

\section{A. Cu cavity}

A copper multicell cavity was fabricated to check the procedures for forming and electron beam welding, and to make sure the desired frequency and field flatness could be obtained. Before field flatness tuning, the accelerating mode frequency of the copper model was $805.9 \mathrm{MHz}$; the initial electric field unflatness parameter $(\Delta E / E)$ was $44 \%$. The field flatness tuning was done using a tuning jig developed for the SNS cavities. The unflatness parameter was $7 \%$ after one iteration of flatness tuning. Thus, the initial frequency error was well within the range of the tuning fixture, and the field flatness tuning was not a problem. The copper model was also used for higher-order mode and input coupling measurements [24].

\section{B. Nb cavity fabrication and preparation}

The results on the copper model being satisfactory, three multicell niobium prototypes were fabricated next. The first 6-cell cavity (Fig. 4) was a simplified version without stiffening rings, dishes for attachment of the helium vessel, or side ports for the rf couplers; these features were included in the second and third cavities (Fig. 5).

Sheet $\mathrm{Nb}$ of thickness $4 \mathrm{~mm}$ with nominal residual resistivity ratio (RRR) of 250 was used for all three cavities. The forming of half-cells and joining were done by the standard deep drawing and electron beam welding techniques used for elliptical cavities, including SNS prototype cavities fabricated at Jefferson Laboratory. As with the SNS cavities, $\mathrm{Nb}$-Ti alloy flanges and $\mathrm{Al}$ alloy gaskets were used for the vacuum seal on the beam tubes. 

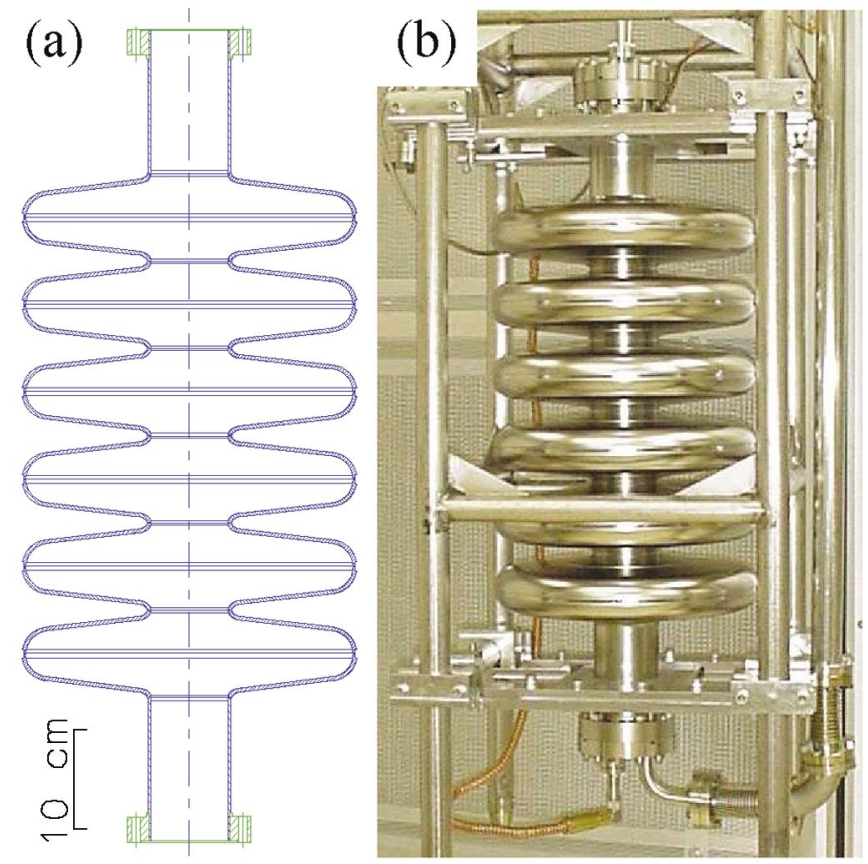

FIG. 4. (Color) (a) Drawing of the first 6-cell $\beta_{g}=0.47$ cavity and (b) photograph of the cavity on the insert. In (a), Nb parts are in blue and $\mathrm{Nb}$ - Ti parts are in green.

The completed 6-cell cavities were etched with 1:1:1 buffered chemical polishing solution (mixture of concentrated phosphoric acid, hydrofluoric acid, and nitric acid in equal parts by volume). About $100 \mu \mathrm{m}$ was removed from
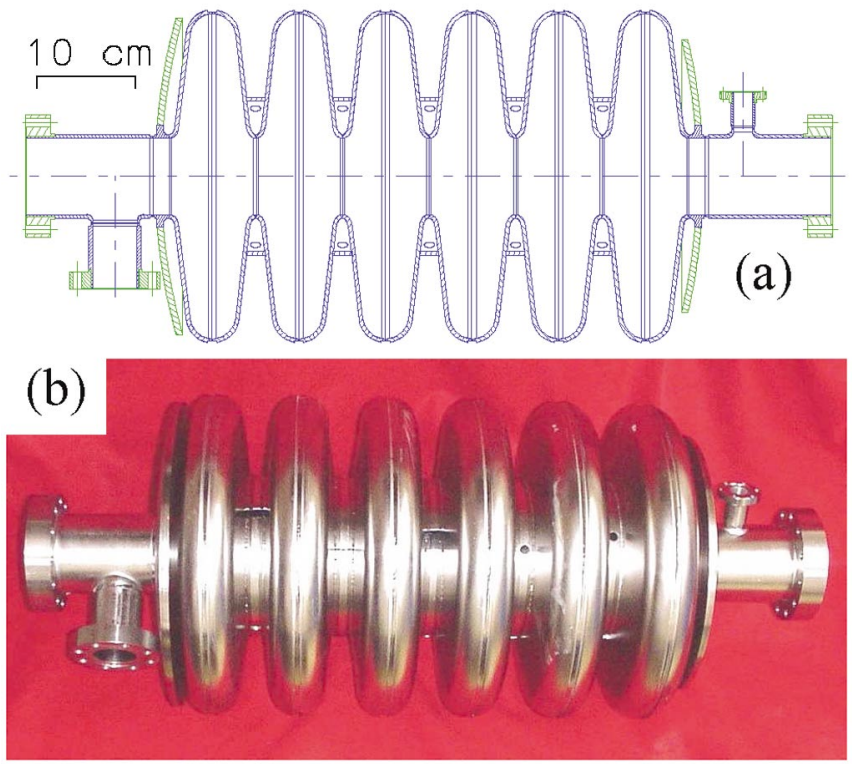

FIG. 5. (Color) (a) Drawing of the second and third 6-cell $\beta_{g}=$ $0.47 \mathrm{Nb}$ cavities and (b) photograph of the second cavity. The port for the input coupler can be seen on the left beam tube. Rings are welded around the outside of the irises to increase the mechanical stiffness of the structure. The end dishes for the attachment of the helium vessel are also visible. the inside surface of the first $\mathrm{Nb}$ multicell. The cavity was then fired in a vacuum furnace for $10 \mathrm{~h}$ at $600{ }^{\circ} \mathrm{C}$ to remove hydrogen and prevent subsequent formation of lossy surface hydrides - in other words, to inoculate it against " $Q$ disease" [26]. The pressure in the furnace was $\leq 10^{-6}$ torr during the heat treatment. Field flatness tuning was done next. The final preparation steps were etching of an additional $60 \mu \mathrm{m}$ from the inner surface and high-pressure rinsing with ultrapure water in a clean room to remove particulates from the inside surface of the cavity. No vacuum bakeout was done after assembly of the cavity onto the insert.

About $150 \mu \mathrm{m}$ was removed during the etch of the second cavity; additional etching was done after the first rf test, as will be described below. About $300 \mu \mathrm{m}$ was removed from the third cavity in 2 etching cycles. The second and third cavities were rinsed with high-pressure water, but no firing was done. The third cavity was rinsed twice, as a leak was found in the input coupler port after the first rinse.

\section{Tuning}

Field flatness tuning was done on all three niobium cavities. The goal was a field unflatness parameter $(\Delta E / E)$ of $10 \%$ or less. The first cavity was tuned with the SNS tuning jig, as had been done with the copper model. After tuning, $\Delta E / E$ was $12 \%$. The tuning was more difficult than it had been with the copper cavity. The second and third $\mathrm{Nb}$ cavities were tuned with a new custom-built jig for the $\beta_{g}=0.47$ cavity. This made the tuning easier; with the second $\mathrm{Nb}$ cavity, $\Delta E / E=5 \%$ was reached in one iteration (see Fig. 6). The results on the third cavity were similar $(\Delta E / E=4.5 \%)$.

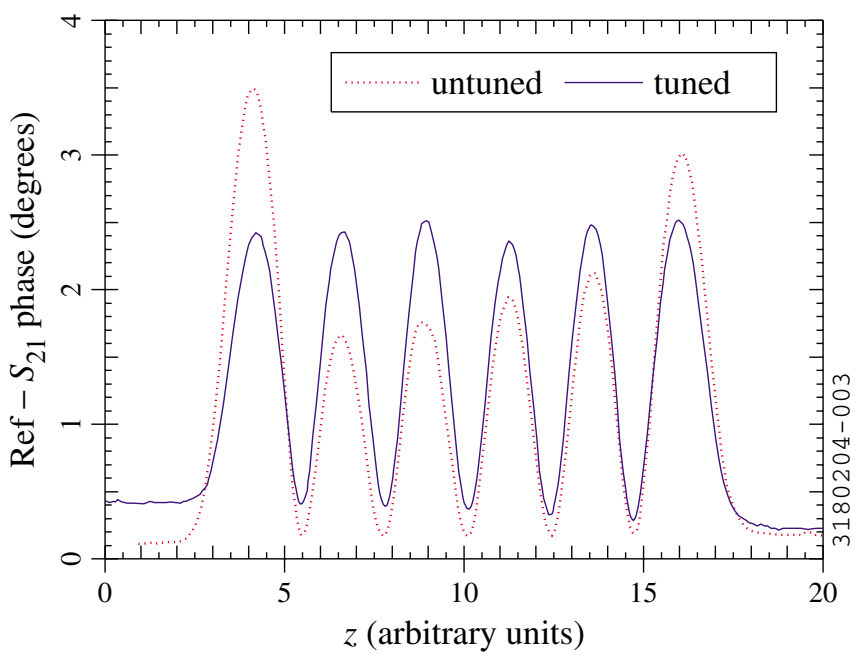

FIG. 6. (Color) Bead pulls for the second 6-cell niobium cavity. The phase shift (proportional to the square of the electric field) as a function of bead position $z$ was measured with a network analyzer. 


\section{RF TESTING OF MULTICELL CAVITIES}

All of the rf tests were done in a vertical cryostat. The residual magnetostatic field inside the cryostat was less than $1 \mu \mathrm{T}$. In all cases, the cryostat was cooled down rapidly to $4.3 \mathrm{~K}$ and then pumped to $2 \mathrm{~K}$. The rf power was supplied by a $500 \mathrm{~W}$ amplifier; a phase feedback loop was used to make sure that the cavity was always driven on resonance. Copper probe antennas on the beam tube end cap flanges (fixed coupling strength) were used for the input coupler and pickup. A radiation sensor on top of the insert was used to monitor $\mathrm{x}$ rays during the rf tests. Radiation shielding surrounded the cryostat during all $\mathrm{rf}$ testing.

\section{A. First test on the first cavity}

The first vertical rf test was done on the first cavity in September 2002. Figure 7 (squares) shows the measurement of the quality factor as a function of field level. As can be seen, the low-field $Q_{0}$ was about $2 \times 10^{10}$ and $Q_{0}$ remained above $10^{10}$ up to $E_{a} \approx 11 \mathrm{MV} / \mathrm{m}$. A gradient of about $16 \mathrm{MV} / \mathrm{m}$ was reached. The test was stopped at that level due to the failure of a rf cable. Some $x$ rays were observed at high field $(0.7 \mathrm{rem} / \mathrm{h})$, indicating that the decrease in $Q_{0}$ at high field was likely due to field emission. Modest rf conditioning (with radiation levels up to $1.2 \mathrm{rem} / \mathrm{h}$ ) was required to reach a gradient of $16 \mathrm{MV} / \mathrm{m}$. A small leak into the cavity vacuum manifested itself when the cryostat was cooled down; the pressure in the cavity was about $10^{-6}$ torr at $2 \mathrm{~K}$.

\section{B. Follow-up tests on the first cavity}

The failed rf cable was replaced, the vacuum leak was fixed, and the cavity was retested 1 week after the first rf

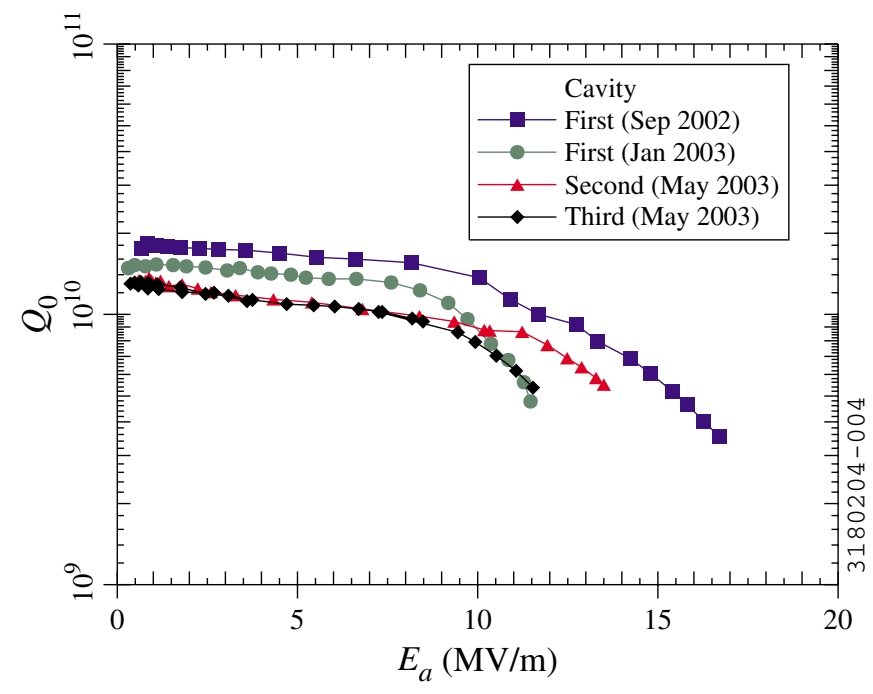

FIG. 7. (Color) Measured dependence of the quality factor on the accelerating gradient for the 6-cell cavity prototypes. All measurements were done at a bath temperature of $2 \mathrm{~K}$. test (without exposure of the inside of the cavity to air). A gradient of about $7 \mathrm{MV} / \mathrm{m}$ was reached. It was thought that helium processing might be beneficial, but the test had to be stopped early due to scheduled maintenance of the cavity testing facility.

The next opportunity for a rf test was in January 2003. In between tests, the cavity was etched to remove another $50 \mu \mathrm{m}$ from the inner surface and the high-pressure water rinsing was repeated. The final filter on the high-pressure rinsing system (between the pump and the nozzle) was temporarily unavailable at the time of this rinse.

The results of the January 2003 test are also shown in Fig. 7 (circles). The low-field $Q_{0}$ was smaller than in the first test, although the difference is within the margin of reproducibility of the measurement. A gradient of about $11 \mathrm{MV} / \mathrm{m}$ was reached. The decrease in $Q_{0}$ between 9 and $11 \mathrm{MV} / \mathrm{m}$ is likely due to field emission; the $\mathrm{x}$-ray signals were larger $(>1.5 \mathrm{rem} / \mathrm{h})$ than those seen in the first $\mathrm{rf}$ test. Thus the difference between the September 2002 and January 2003 tests could be due to particulate contamination during the high-pressure rinse without the final filter. Although the field level was not as high as in the first test, $Q_{0}$ was nevertheless in excess of $10^{10}$ at $E_{a}=8 \mathrm{MV} / \mathrm{m}$.

\section{Tests on the second cavity}

The second cavity was tested in May 2003. In the first rf test, a gradient of $8 \mathrm{MV} / \mathrm{m}$ was reached at $2 \mathrm{~K}$. The $Q_{0}$ was lower $\left(8 \times 10^{9}\right)$ and some $Q$ switching was seen, indicating that more etching was needed.

In preparation for a second rf test, another $150 \mu \mathrm{m}$ was removed from the inner surface, and the high-pressure rinse was repeated. Results from the second test are shown in Fig. 7 (triangles). A gradient of $13 \mathrm{MV} / \mathrm{m}$ was reached. The $Q_{0}$ was $10^{10}$ at $E_{a}=8 \mathrm{MV} / \mathrm{m}$. The field was limited by the available rf power (the input coupling was weaker than planned). The $\mathrm{x}$-ray signals were small $(10 \mathrm{mrem} / \mathrm{h}$ maximum).

\section{Tests on the third cavity}

The third cavity was also tested in May 2003. Results are shown in Fig. 7 (diamonds). A gradient of $11 \mathrm{MV} / \mathrm{m}$ was reached. The $Q_{0}$ was again about $10^{10}$ at $E_{a}=8 \mathrm{MV} / \mathrm{m}$. The field was again limited by the available rf power (the same weak input antenna was used). As with the second cavity, the x-ray signals were small $(0.5 \mathrm{mrem} / \mathrm{h}$ maximum).

\section{E. Temperature dependence}

Small $\mathrm{rf}$ losses in the cavity are desirable to minimize the load to the cryogenic system. Low rf losses correspond to a large $Q_{0}$, or, equivalently, a small rf surface resistance $R_{s}$, since $Q_{0}=G / R_{s}$, where $G$ is the geometry factor. An approximate expression for the surface resistance of $\mathrm{Nb}$ is [26] 


$$
R_{s}(T)=R_{0}+C_{\mathrm{RRR}} R_{1} \frac{T_{\Delta}}{T}\left(\frac{f}{f_{1}}\right)^{2} \exp \left(-\frac{T_{\Delta}}{T}\right),
$$

where $T$ is the temperature, $f$ is the $\mathrm{rf}$ frequency, $T_{\Delta}=$ $17.67 \mathrm{~K}, R_{1} T_{\Delta}=2 \times 10^{-4} \Omega \mathrm{K}$, and $f_{1}=1.5 \mathrm{GHz} ; R_{0}$ is the temperature-independent residual surface resistance. The coefficient $C_{\mathrm{RRR}}$ is 1 for reactor grade $\mathrm{Nb}$ (RRR $=$ 25 ) and about 1.5 for high purity $\mathrm{Nb}$ with $\mathrm{RRR}=250$. As the surface purity can be different from the bulk purity, $C_{\mathrm{RRR}}$ can be considered to be a fitting parameter. Equation (1) is valid for $f \ll 1 \mathrm{THz}$ and $T \leq 4.6 \mathrm{~K}$.

Figure 8 shows the measured rf surface resistance at low field $\left(E_{a} \leq 0.8 \mathrm{MV} / \mathrm{m}\right)$ as a function of $1 / T$. The solid lines are calculated from Eq. (1), assuming $C_{\mathrm{RRR}}=1.25$ and the indicated $R_{0}$ values. In all cases, the surface resistance at higher temperatures follows Eq. (1) reasonably well. This suggests that the surface RRR is smaller than the nominal bulk value of 250 , but higher than that of reactor grade $\mathrm{Nb}$. The $R_{s}$ values at low temperatures are consistent with $R_{0}$ being between 2 and $9 \mathrm{n} \Omega$.

The results for the single-cell cavities were similar to those of the 6-cell cavities. In the best single-cell tests, the temperature-dependent losses were slightly lower than for the 6-cell cavities.

In the January 2003 test on the first 6-cell cavity, measurements of $Q_{0}$ as a function of gradient were done at several different temperatures, as shown in Fig. 9. A significant downward slope in $Q_{0}$ can be seen for the measurements above the superfluid transition temperature $(2.15 \mathrm{~K})$, as is typically seen. Thus, high-field operation of the cavities is more economical for $T<2.15 \mathrm{~K}$, as one would expect. The low-field $Q_{0}$ at $1.8 \mathrm{~K}$ is significantly higher than at $2 \mathrm{~K}$, which confirms that the temperaturedependent losses are still contributing to the surface resist-

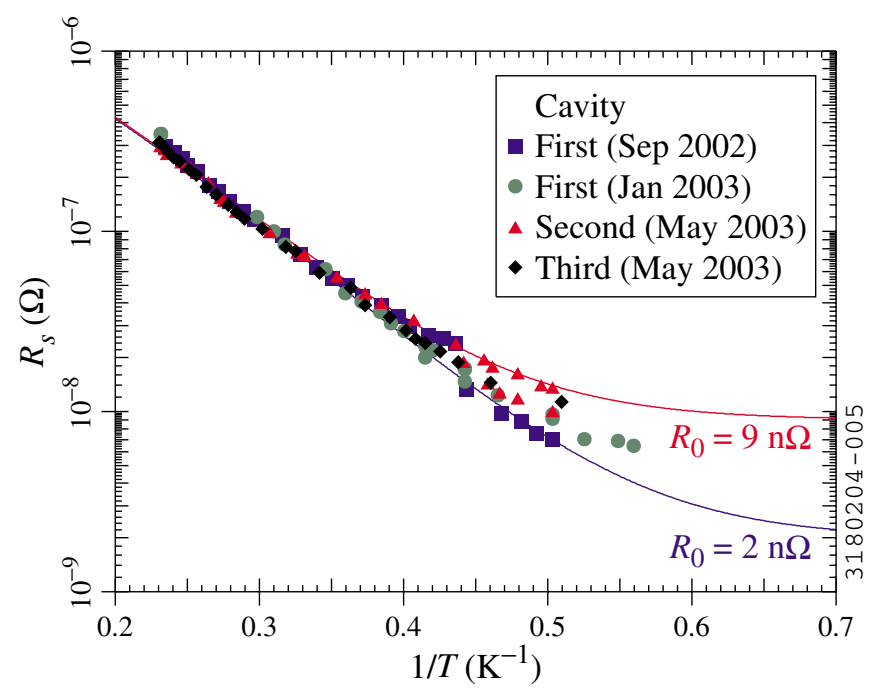

FIG. 8. (Color) Measured dependence of the rf surface resistance on bath temperature for the 6-cell cavity prototypes. The solid lines are theoretical predictions for $C_{\mathrm{RRR}}=1.25$ and $R_{0}=$ 2 or $9 \mathrm{n} \Omega$.

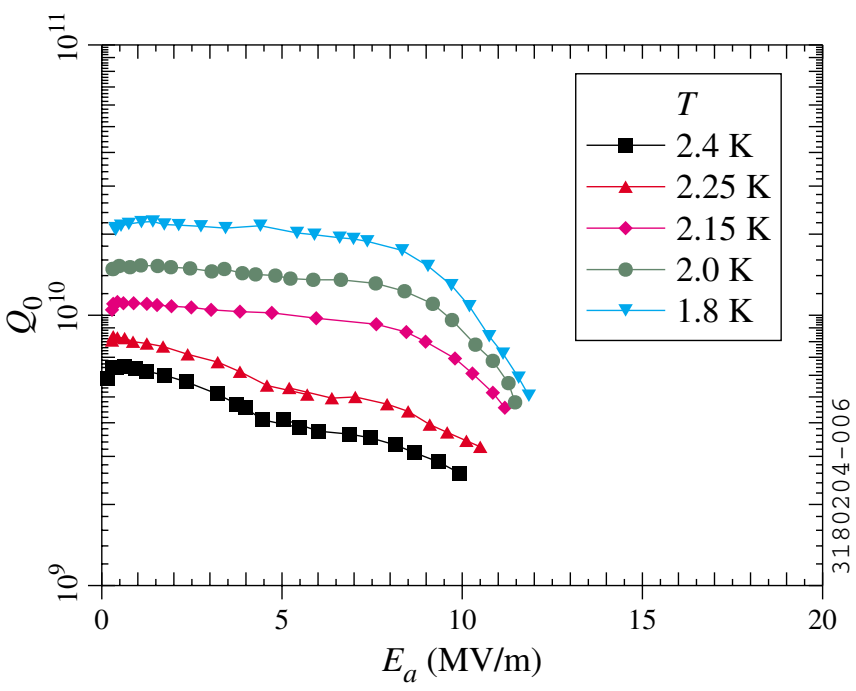

FIG. 9. (Color) Measured dependence of the quality factor on the accelerating gradient at different bath temperatures for the first 6-cell cavity prototype (January 2003).

ance. However, the maximum gradient at $1.8 \mathrm{~K}$ is only slightly higher $(11.4 \mathrm{MV} / \mathrm{m})$ than at $2 \mathrm{~K}$.

\section{MICROPHONICS AND MULTIPACTING}

Microphonics are more serious for RIA than for SNS due to the lower RIA beam current. The stiffening rings of the SNS cavities will be used on the $\beta_{g}=0.47$ cavity to reduce microphonic excitation (see Fig. 5 above). Lateral bracing similar to the center support "spider" of the SNS cavities $[11,27]$ will also be implemented. The RIA cavities will be overcoupled to ensure that the gradient can be maintained in the presence of microphonics [24]. Some microphonic measurements were done on a single-cell cavity [18]. Modeling of vibrations in multicell cavities is in progress. The predictions will be compared with measurements on the 6-cell cavities.

The rf tests on single-cell cavities showed that there are no hard multipacting barriers. A soft multipacting barrier was seen occasionally at very low field $\left(E_{a}<1 \mathrm{MV} / \mathrm{m}\right)$. After we introduced gas for He processing, the barrier became more persistent, but we could still punch through it. Multipacting simulations $[20,28]$ also indicate that there should be no hard barriers in the single-cell cavities. Likewise, no multipacting problems were encountered in the rf tests on the multicell cavities.

\section{CONCLUSION}

Encouraging rf test results were obtained for two singlecell $\beta_{g}=0.47$ cavity prototypes and three 6 -cell cavities: all of the cavities exceeded the goal of $10 \mathrm{MV} / \mathrm{m}$ accelerating gradient, with $Q_{0} \geq 7 \times 10^{9}$ at $E_{a}=10 \mathrm{MV} / \mathrm{m}$ and $Q_{0} \geq 10^{10}$ at $E_{a}=8 \mathrm{MV} / \mathrm{m}$. The first 6-cell cavity and both single-cell cavities reached $E_{a} \geq 16 \mathrm{MV} / \mathrm{m}$; the 
second and third 6-cell reached 13 and $11 \mathrm{MV} / \mathrm{m}$, respectively. The three niobium multicells and one copper multicell have been tuned for field flatness. The next step will be a horizontal test of 2 fully equipped $\beta_{g}=0.47$ cavities in a prototype cryomodule [29]. Because the input coupling and HOM damping requirements are less stringent, the system will be less complicated than for SNS.

\section{ACKNOWLEDGMENTS}

We thank the staff at INFN-Milano, Jefferson Lab, and MSU for their hard work in the design, fabrication, processing, and testing of the cavity prototypes. R. Afanador, J. Brawley, B. Manus, S. Manning, S. Morgan, G. Slack, and L. Turlington provided essential support with the fabrication and chemical treatment of the cavities at Jefferson Lab. J. Bierwagen, J. Brandon, S. Bricker, J. Colthorp, S. Hitchcock, M. Johnson, H. Laumer, D. Lawton, A. McCartney, D. Pedtke, L. Saxton, J. Vincent, and R. Zink provided valuable support at MSU. This work was supported by the U.S. Department of Energy under Grant No. DE-FG02-03ER41247.

[1] H.A. Thiessen, in Proceedings of the 1991 Particle Accelerator Conference, San Francisco, CA, edited by L. Lizama and J. Chew (IEEE, Piscataway, NJ, 1991), pp. 3198-3200.

[2] W. B. Haynes et al., in Proceedings of the Eighth Workshop on RF Superconductivity, Abano Terme, Italy, 1997, edited by V. Palmieri and A. Lombardi (LNL-INFN Report No. 133/98), pp. 523-533.

[3] K. Saito et al., in Proceedings of the Eighth Workshop on RF Superconductivity, Abano Terme, 1997 (Ref. [2]), pp. 534-539.

[4] N. Ouchi et al., IEEE Trans. Appl. Supercond. 9, 1030 (1999).

[5] J. L. Biarrotte et al., in Proceedings of the 9th Workshop on RF Superconductivity, Los Alamos, NM, 1999 (LANL Report No. LA-13782-C, 2000), pp. 384-389.

[6] S. Bauer et al., in Proceedings of the 9th Workshop on RF Superconductivity, Los Alamos, NM, 1999 (Ref. [5]), pp. 443-445.

[7] N. Akaoka et al., in Proceedings of the 9th Workshop on RF Superconductivity, Los Alamos, NM, 1999 (Ref. [5]), pp. $450-458$.

[8] E. Chiaveri et al., in Proceedings of the 9th Workshop on RF Superconductivity, Los Alamos, NM, 1999 (Ref. [5]), pp. 512-515.

[9] T. Tajima et al., in Proceedings of the 2001 Particle Accelerator Conference, Chicago, IL, edited by P. Lucas and S. Webber (IEEE, Piscataway, NJ, 2001), pp. 11191121.

[10] C. Pagani et al., in Proceedings of the 2001 Particle Accelerator Conference, Chicago, IL (Ref. [9]), pp. 3612-3614.
[11] G. Ciovati et al., in Proceedings of the European Particle Accelerator Conference (EPAC 2002), Paris, 2002, edited by T. Garvey et al. (EPS-IGA and CERN, Geneva, 2002), pp. 2247-2249.

[12] M. Mizumoto et al., in Proceedings of the $X X$ International Linac Conference, Monterey, CA, edited by A. W. Chao (SLAC Report No. SLAC-R-561, 2000), pp. $566-568$.

[13] C. W. Leemann, in Proceedings of the XX International Linac Conference, Monterey, CA (Ref. [12]), pp. 331-335.

[14] K. W. Shepard et al., in Proceedings of the 9th Workshop on RF Superconductivity, Los Alamos, NM, 1999 (Ref. [5]), pp. 345-351.

[15] K. C. D. Chan et al., in Proceedings of the 9th Workshop on RF Superconductivity, Los Alamos, NM, 1999 (Ref. [5]), pp. 465-471.

[16] A. Facco, in Proceedings of the 11th Workshop on RF Superconductivity, Travemünde, Germany, 2003 (DESY, Hamburg, 2004).

[17] C. C. Compton et al., in Proceedings of the 2001 Particle Accelerator Conference, Chicago, IL (Ref. [9]), pp. 10441046.

[18] T. Grimm et al., in Proceedings of the Tenth Workshop on RF Superconductivity, Tsukuba, 2001, edited by S. Noguchi (KEK, Tsukuba, Japan, 2003), pp. 86-90.

[19] W. Hartung et al., in Proceedings of the 2003 Particle Accelerator Conference, Portland, OR, edited by J. Chew, P. Lucas, and S. Webber (IEEE, Piscataway, NJ, 2003), pp. 1362-1364.

[20] D. Barni et al., Jefferson Laboratory Technical Note No. JLab-TN-01-014, 2001.

[21] B. Aune et al., Phys. Rev. ST Accel. Beams 3, 092001 (2000).

[22] K. Halbach and R.F. Holsinger, Part. Accel. 7, 213 (1976).

[23] J.H. Billen and L. M. Young, in Proceedings of the 1993 Particle Accelerator Conference, Washington, DC, edited

S. T. Corneliussen (IEEE, Piscataway, NJ, 1993), pp. 790-792.

[24] T.L. Grimm et al., in Proceedings of the European Particle Accelerator Conference (EPAC 2002), Paris, 2002 (Ref. [11]), pp. 2241-2243.

[25] D. G. Myakishev and V. P. Yakovlev, in Proceedings of the 1991 Particle Accelerator Conference, San Francisco, CA (Ref. [1]), pp. 3002-3004.

[26] H. Padamsee, J. Knobloch, and T. Hays, $R F$ Superconductivity for Accelerators (Wiley, New York, 1998).

[27] J. Hogan et al., in Proceedings of the 2001 Particle Accelerator Conference, Chicago, IL (Ref. [9]), pp. 1158-1159.

[28] W. Hartung, F. Krawczyk, and H. Padamsee, in Proceedings of the Tenth Workshop on RF Superconductivity, Tsukuba, 2001 (Ref. [18]), pp. 627631.

[29] T.L. Grimm et al., in Proceedings of the 2003 Particle Accelerator Conference, Portland, OR (Ref. [19]), pp. 1350-1352. 